\title{
CONVENTIONAL AND PULSE CATHODIC PROTECTION OF REINFORCED CONCRETE: ELECTROCHEMICAL APPROACH AND MICROSTRUCTURAL INVESTIGATION
}

\author{
D.A.Koleva ${ }^{\mathrm{a}}$, J.Hu ${ }^{\mathrm{a}}$, K.van Breugel ${ }^{\mathrm{a}}$, J.H.W.de $\mathrm{Wit}^{\mathrm{b}}$ and N.Boshkov ${ }^{\mathrm{c}}$ \\ ${ }^{a}$ Delft University of Technology, CiTG, Stevinweg 1, 2628 CN, Delft, NL \\ ${ }^{\mathrm{b}}$ Delft University of Technology, GvTM, Rotterdamseweg 137, 2628 AL Delft, NL \\ ${ }^{\mathrm{c}}$ Bulgarian Academy of Sciences, IPC, G.Bonchev bl.11, Sofia, BG
}

An improved cathodic protection (CP), based on pulse regime was investigated in reinforced concrete, compared to conventional $\mathrm{CP}$. Both regimes used $\mathrm{CP}$ current in the range of 2 to $15 \mathrm{~mA} / \mathrm{m}^{2}$. A voltage-to-current converter maintained the pulse current, the latter adjusted with duty cycle of $12 \%$ to $50 \%$ and frequency of $500 \mathrm{~Hz}$ to $1 \mathrm{kHz}$. The efficiency of the improved CP was evaluated by means of electrochemical methods and microstructural investigation of the bulk concrete matrix, the steel/paste interface and the steel surface. The combination of techniques provides a detailed understanding of the electrochemical phenomena associated with corrosion and protection processes. The pulse CP achieves sufficient protection of the steel reinforcement and is less detrimental to the bulk concrete matrix. In this sense, pulse CP is able to minimize the negative side effects and to ensure better performance and protection in reinforced concrete.

\section{INTRODUCTION}

Corrosion of reinforcing steel in concrete structures as result of environmental influences and degradation due to aggressive substances represents a great concern with respect to infrastructure durability and sustainability. The prevention and protection techniques based on electrochemical principles and methods for monitoring and control are of significant importance, interest and applicability to those systems. This study presents part of the research outcomes from an extensive research on establishing an improved impressed current CP (ICCP) system, based on pulse technology. The research combines investigation of fundamental behavior of materials and adjusting optimum regime and parameters for the performance of the steel reinforcement/concrete system, thus correlating micro-level research with engineering level applications.

Generally, the steel in hardened concrete is protected by a passive layer which remains stable in the highly alkaline concrete medium. However, when sufficient aggressive ions (e.g., sulphate and chloride from seawater or chloride from de-icing salts) have penetrated to the reinforcement or when the $\mathrm{pH}$ of the pore solution drops to low values due to carbonation, the protective film is destroyed and corrosion initiated. Cathodic protection (CP) has been found to be the only proven method and working technique to stop corrosion in reinforced concrete (1). In this study, the polarization of the steel reinforcement is achieved by supplying impressed direct current (DC) - steady and 
pulse, to the steel embedded in concrete specimens. The repulsion of aggressive anions (e.g. chloride) which takes place along with the protection itself is a beneficial one as far as corrosion risk of the steel is concerned. Along with ion migration and diffusion due to the $\mathrm{CP}$ current, the heterogeneities and instabilities inherent in the concrete material may lead to non-uniform distribution of the current and thus result in localized overprotected areas. The effect of concrete resistivity and applied current density are of utmost importance for the proper distribution of electrical current in a reinforced concrete $\mathrm{CP}$ system. Furthermore, concrete resistance increases with aging. As reported in (2), increasing both the applied current density and concrete resistivity leads to worse current distribution in the system. As previously investigated by the authors (3), unfavorable pore structure alterations in cement-based materials yield non-uniform electrical properties and thereby possibly result in disturbance of the electrolytic path (i.e. the current demand is increasing). In addition, CP current induces structural alterations in the bulk concrete and in the pore space, causes micro-cracking which along with softening of the C-S-H leads to reduced concrete durability, as reported also in (4). The main negative effect in terms of concrete microstructure is enlarging the gap between cement paste and aggregate in the interfacial transition zones (ITZs), thus yielding micro-cracks. These unfavorable microstructure alterations can lead to concrete degradation. In CP applications, electrical properties are additionally influenced by the current flow through the concrete bulk matrix and as also reported for other electrochemical (rehabilitation) techniques (5), electrical current and chemical binding/de-binding mechanisms strongly influence the bulk matrix properties, leading to non-uniform ion migration and current distribution. This constitutes an additional feature that should be overcome in engineering application of the CP techniques. Moreover, as reported elsewhere $(6,7)$ an excessive protection current will cause increased hydrogen evolution reactions on the steel surface and lead to hydrogen embrittlement in case of prestressed steel reinforcement and steel/cement paste bond degradation.

The above mentioned and well-known side effects of the impressed current $\mathrm{CP}$ technique can be possibly overcome by establishing a certain current regime based on pulse current, which achieves sufficient protection of the steel reinforcement, while at the same time, minimizes the influence of current flow on micro-level and avoids overprotection. This research investigates the feasibility and efficiency of Pulse ICCP applied to reinforced concrete and its superiorities over conventional CP. The study explores the correlation of widely used techniques for evaluation of $\mathrm{CP}$ efficiency, mainly based on empirical criteria, with laboratory techniques as Linear Polarization resistance (LPR), Electrochemical Impedance Spectroscopy (EIS), additionally combining the latter with SEM observation of the concrete bulk matrix and the corroding and protected steel surface, thus evaluating the efficiency of the improved pulse $\mathrm{CP}$ technique in a fundamental approach in combination with generally accepted methods.

\section{EXPERIMENTAL}

Materials. The present study combines investigation on two series of specimens in terms of evaluating the influence of technical conditions and CP prevention/protection techniques on both materials - steel and concrete. Concrete prisms and reinforced concrete cylinders were cast using OPC CEM I 32.5, cement-sand-gravel ratio 1:2:4, w/c ratio 0.6 , curing conditions $14(28)$ days fog room $(98 \% \mathrm{RH}, 20 \mathrm{EC})$, climate room at $50 \%$ $\mathrm{RH}$ (for the prisms) and certain lab environment (for the cylinders) afterwards. 
Concrete prisms (Fig.1a): Dimensions 100x100x300mm, differing in chemical composition and set-up. Subgroups: D - cast using demi-water, N - cast using $1.25 \mathrm{M}$ $\mathrm{NaCl}$ solution as mixing water, $\mathrm{DN}$ - half-to-half the mixture of $\mathrm{D}$ and $\mathrm{N}$. The series of concrete prisms was investigated aiming at determination of electrical properties in all technical conditions and correlating results with alterations in chemical composition (chlorides and alkali concentrations).

Reinforced concrete cylinders (Fig. $1 \mathrm{~b}$ ): Dimensions $\mathrm{H}=25 \mathrm{~cm}, \mathrm{D}=12 \mathrm{~cm}$, containing 2 embedded construction steel bars $(\mathrm{d}=12 \mathrm{~mm})$. Specimens were conditioned in salt spray chamber $(5 \% \mathrm{NaCl})$ for certain period of the test, aiming at acceleration of the corrosion process. Subgroups: N-corroding cells, P-protected cells (pulse and conventional CP applied), R non-corroding cells (control specimens). All cells contained embedded reference electrode $\left(\mathrm{Mn} / \mathrm{MnO}_{2}\right)$ and Mixed Metal Oxide (MMO) Ti mesh on the outer surface (serving as counter electrode or anode). This series aimed at investigation the efficiency of different regimes of cathodic protection, as well as the electrochemical behavior of the embedded steel. Cathodic protection (conventional and pulse) was applied at 30 days of cement hydration for part of the specimens, using $2 \mathrm{~mA} / \mathrm{m}^{2}$, aiming at investigation of the technique as prevention method. The rest of the specimens from group $\mathrm{P}$ were under protection starting at 60 days of cement hydration, using steady and pulse DC in different regimes (from 5 to $20 \mathrm{~mA} / \mathrm{m}^{2}$ applied on different cells for the whole test period and from 12 to $50 \%$ duty cycle at $500 \mathrm{~Hz}$ to $1 \mathrm{kHz}$ for the pulse regime).
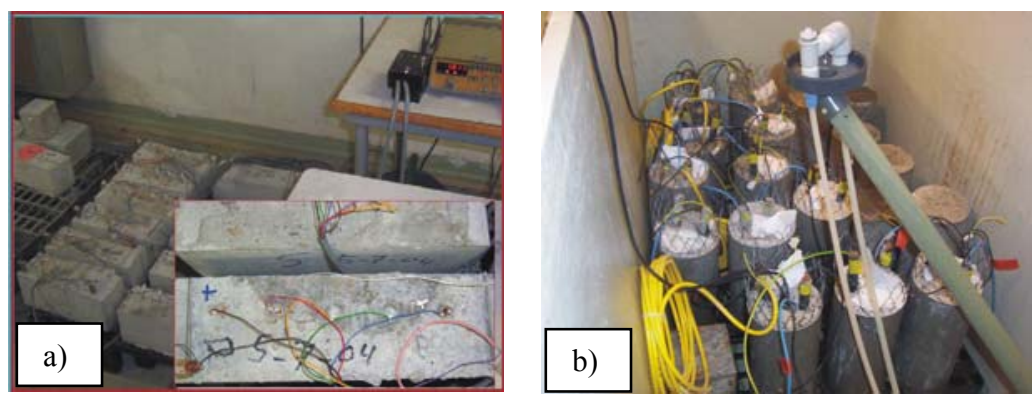

FIGURE 1. Experimental set-up: a) Concrete prisms; b) Reinforced concrete cylinders

Experimental techniques. With regard to evaluation of the influence of $\mathrm{CP}$ current on chemical composition and structural properties of cement-based materials, the series of concrete prisms was under electrical resistivity monitoring and chemical analysis for chloride and alkali concentrations. The electrical resistivity (by sections of the prisms and in total) was calculated on basis of recorded resistance. An AC 4 pin method was performed using specially designed R-meter via embedded plates (on the prisms cross section edges), pins and multiplexers, thus it was possible to select and record electrical resistance in a certain cell or cell section. The chemical analysis was performed for each section of the specimens at certain intervals of time (from 14 to 210 days of cement hydration), according ASTMC1218 and ASTM C1152 for free and total chloride concentration and using plasma spectrometry (Inductive Coupled Plasma Spectrometer (ICP-AES) for determination of alkali concentrations. The chemical analysis aimed at monitoring ion transport due to diffusion (no-current conditions) and migration under electrical field (DC and pulse DC current conditions).

The reinforced concrete specimens were maintained in different technical conditions, aiming at acceleration corrosion process on the steel surface first and applying cathodic protection afterwards (both conventional and pulse) at certain intervals (generally starting 
at 60 days of cement hydration). The monitoring techniques included generally accepted Half-cell potential mapping (according ASTMC876) and Polarization Development/Decay (according NACE RP0290-2000). In addition electrochemical methods - Linear Polarization Resistance (LPR), Potentiodynamic Polarization (PD) and Electrochemical Impedance Spectroscopy (EIS) were performed. All electrochemical methods aimed at investigation of electrochemical parameters for the steel surface, mainly determination of the Polarization resistance $(\mathrm{Rp})$ in every technical condition. Electrochemical methods were performed at OCP for all cells (after $24 \mathrm{~h}$ depolarization of the reinforcement for the protected specimens). LPR applied an external polarization in the range of $\pm 20 \mathrm{mV}$ vs OCP, scan rate $0.16 \mathrm{mV} / \mathrm{s}$, PD was performed in the range of $0.15 \mathrm{~V}$ to $+1.2 \mathrm{~V}$ vs OCP. The EIS measurements were carried out in the frequency range $50 \mathrm{kHz}$ to $10 \mathrm{mHz}$ by superimposing an $\mathrm{AC}$ voltage of $10 \mathrm{mV}$. All electrochemical measurements were performed when the reinforced concrete cylinders were in immersed condition $(5 \% \mathrm{NaCl}$ solution for corroding and protected specimens and demi-water for non-corroding specimens), assuring electrical conductivity of the medium. The used equipment was EcoChemie Autolab - Potentiostat PGSTAT30, combined with FRA2 module, using GPES and FRA software package.

Relevant to investigation of the bulk concrete material in every technical condition, section images of the specimens were obtained by scanning electronic microscopy (SEM) with backscattered electrons mode (BSE). A set of SEM images were made at random locations on polished sections, the physical size of the reference region of each image is $226 \mu \mathrm{m}$ in length and $154 \mu \mathrm{m}$ in width, with the resolution of $0.317 \mu \mathrm{m} / \mathrm{pixel}$ (corresponding to a magnification of 500x). The SEM micrographs were subject to quantitative image analysis for derivation of structural parameters. In addition, morphological aspects and chemical compositions of products, associated with corrosion and cathodic protection, were investigated as well. For this purpose, environmental SEM Philips XL30 equipped with energy dispersive X-ray analysis (EDXA) is used for morphological and microstructural investigations of the corrosion products in unprotected (corroding group) and protected (under $\mathrm{CP}$ and pulse $\mathrm{CP}$ ) specimens.

\section{RESULTS AND DISCUSSION}

\section{Electrical properties and chloride ions transport in $\mathrm{CP}$ conditions.}

Electrical measurements included monitoring of electrical resistivity by sections of the concrete prisms and in total, recording alterations due to hydration mechanisms, current flow (both steady and pulse DC) and ion transport due to diffusion and migration. Resistance monitoring was performed during a period of 210 days of cement hydration. The total electrical resistivity and local resistivity at various sections are calculated using the equation: $\rho=R A / l$, where $\rho$ is the resistivity (in Ohm-cm), $R$ is the resistance (in Ohm), $A$ is the cross section area (in $\mathrm{cm}^{2}$ ) and $l$ is the length (in $\mathrm{cm}$ ). Figs. 2 a-c) depict the evolution of total electrical resistivity for all groups in different technical conditions, i.e. in rest conditions (only cement hydration is involved), under pulse and conventional CP current with the current flowing through the cell as an additional influencing factor.

The electrical current was applied after 30 days of cement hydration to part of the concrete specimens (simulating CP current) and aimed at investigating the relationships 
between electrical properties and microstructure changes induced by the current flow. Since 60 days of cement hydration, all specimens were placed in climate room $(50 \% \mathrm{RH})$.

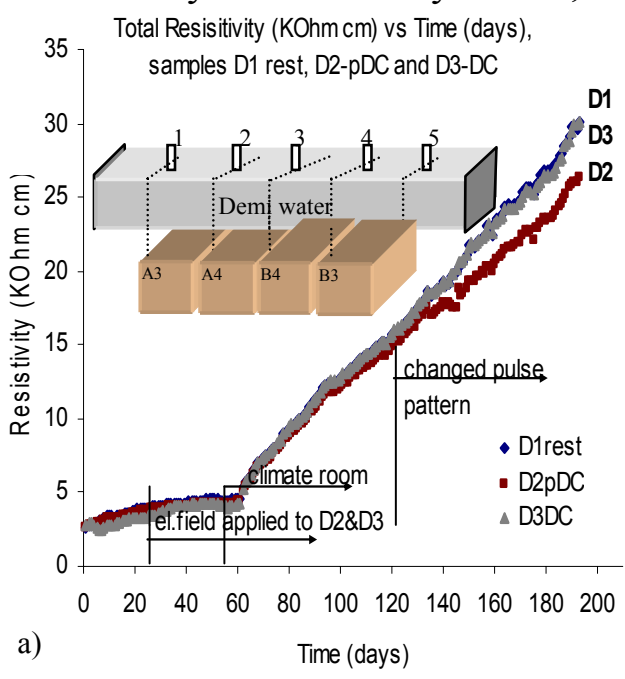

Total Resisitivity (KOhm cm) vs Time (days), samples DN1, DN2-pDC, DN3-DC

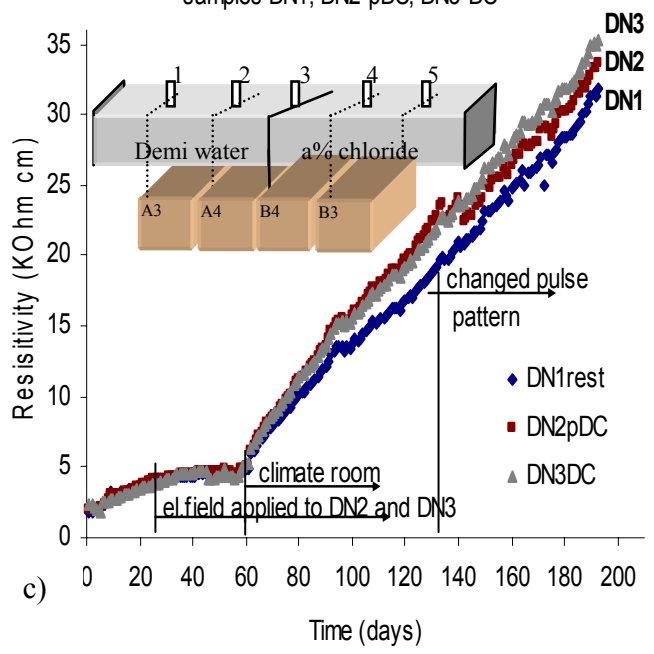

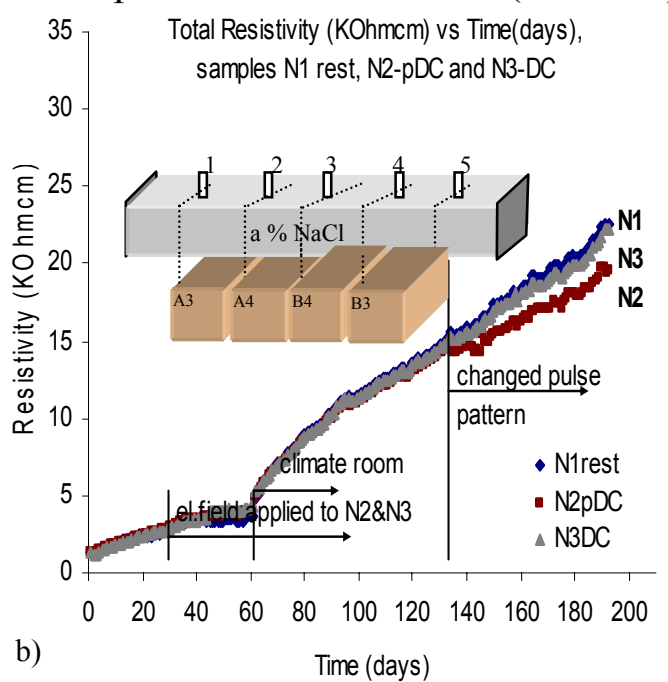

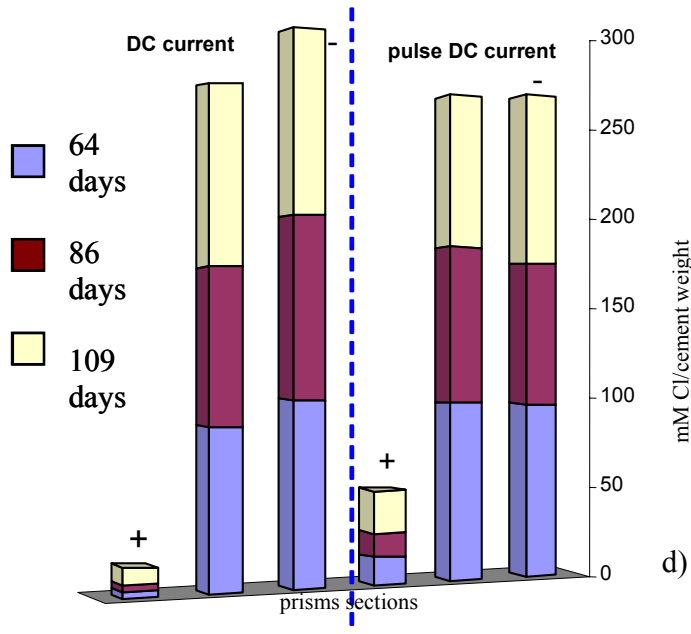

FIGURE 2 Electrical resistivity vs time (at 210 days of age) in every technical condition and chloride concentrations (cumulative up to 109 days of age): (a) group D (using demi- water); (b) group $\mathrm{N}$ (using 1.25M NaCl); (c) group DN (half-to-half the mixture of $\mathrm{D}$ and $\mathrm{N}$ ); (d) chloride concentration (mMol per cement weight) for group DN in conditions of steady and pulse DC.

As expected the electrical resistivity shows a considerable shift towards higher values as specimens were drying out. The influences of DC current flow on electrical properties for specimens $\mathrm{D}$ and $\mathrm{N}$ are minor, while specimens $\mathrm{DN}$ show a notable increase in electrical resistivity due to the applied current (Fig. 2c). The reason is that the $\mathrm{Cl}^{-}$ concentration gradient in the DN specimen additionally promotes ion transport and accelerates the hydration process. Changing the pulse pattern at about 90 days lead to decline in the resistivity for pulse current conditions for all specimens, which is in favor of the concrete microstructure, since lower resistivity will ease ion and current flow in the concrete systems.

When chloride ions penetrate through the concrete surface and ingress in the bulk material, part of them undergo chemical binding, part of them remain physically bound, 
and the others stay free in the pore solution. Bound chlorides can be released when carbonation takes place (8). In case of CP applications, ion transport is induced by the current flow and as far as transport of chlorides is concerned, it has a beneficial effect on the global performance of the system, since chlorides are withdrawn away from the negative pole (the reinforcement being negatively polarized in $\mathrm{CP}$ applications). In the specimens under CP current regimes (steady and pulse DC), the current flow is accelerating the ion transport towards sections A (initially free of chloride - see schemes in Figs.2b,c), where the ion-exchange capacity is higher, thus increasing the hydration rate and filling up of the pore system. In the case of pulse $\mathrm{CP}$, the physicochemical process is taking place more uniformly throughout the specimens than the specimens under steady DC, evidenced by the local resistivity measurements (Figs. 2). This effect can be additionally proved by the lower and almost uniform chloride concentrations in specimen under pulse DC (Fig.2d) at the middle part and negatively charged pole of the specimens, accompanied by a higher chloride concentration around the positively charged pole, compared to the specimens under steady DC current. Hence, pulse DC current is more efficient in enhancing the chloride migration towards the anode and reducing the aggressive ion concentration around the cathode (Fig.2d).

\section{Half Cell potential mapping and Depolarization measurements.}

Potential mapping does not determine corrosion rate and protection efficiency, but estimates the probability of corrosion. With respect to $\mathrm{CP}$ efficiency, potential reading determines if the electrode potential falls in the region of "immunity" (according generally accepted criteria and for e.g. Pourbaix diagrams). In the present study, potential mapping was performed in accordance with ASTM C876, using embedded $\mathrm{Mn} / \mathrm{MnO}_{2}$ reference electrodes and additional comparative measurement with external SCE electrode. Fig. 3a) depicts the recorded values vs SCE for the period of 240 days.

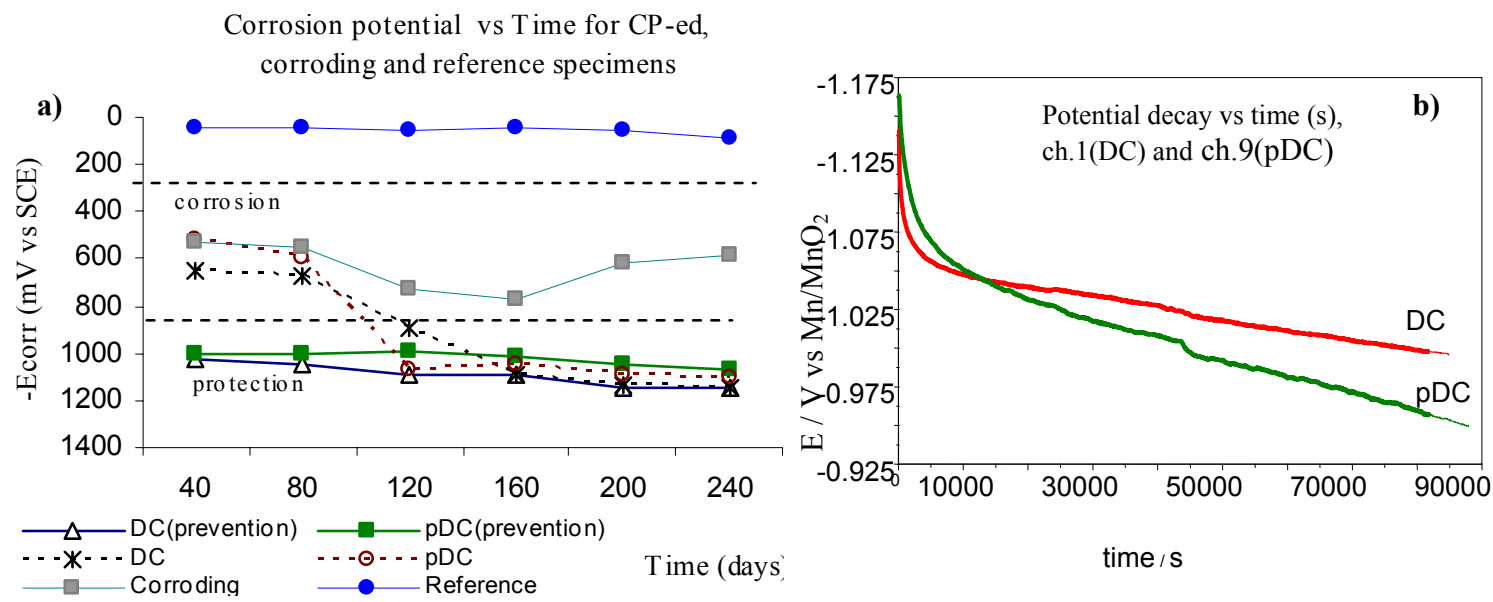

FIGURE 3 Potential mapping for corroding, non-corroding (control reference) and protected (conventional and pulse $\mathrm{CP}$ ) specimens - (a) and Depolarization /Decay measurements for specimens under conventional and pulse CP - (b).

Fig.3b) presents a depolarization (decay) measurement, performed according generally accepted criteria (e.g. NACE RP0290-2000). The decay was determined by interrupting the $\mathrm{CP}$ current and monitoring the potential of the steel bars, relative to embedded (and alternatively external) reference electrodes $\left(\mathrm{Mn} / \mathrm{MnO}_{2}\right.$ and SCE). As initial value the "instant-off" potential is used, the decay according criteria should be at 
least $100 \mathrm{mV}$ for $4 \mathrm{~h}(24 \mathrm{~h})$, which determines effective CP. All measurements were performed in equally controlled for all cells environmental conditions.

\section{Electrochemical methods}

3.1. Electrochemical Impedance Spectroscopy (EIS). The technique allows retrieving information for the electrochemical behavior of the steel surface and determination of the bulk matrix properties as well. Thus, the global system performance can be evaluated, taking into consideration both materials - concrete and steel. For the present study EIS was performed aiming at retrieving the polarization resistance $\mathrm{Rp}$ of the steel bars. More detailed information for the efficiency of the technique, physical meaning and related considerations with regard to steel in cement-based materials can be referred to $(9,10)$ and is reported by the present authors in (11) as well. EIS measurements were performed at OCP (after depolarization of $24 \mathrm{~h}$ for the protected specimens) by superimposing an AC voltage of $10 \mathrm{mV}$ in the frequency range of $50 \mathrm{kHz}$ to $10 \mathrm{mHz}$. The equivalent el. circuit on Fig.4b) was used to fit the experimental data (Figs.5a,b). The elements from the equivalent electrical circuit are attributed to definite physical parameters, corresponding to certain interfaces (Fig.4a) in the reinforced concrete system. A simplified definition of the model is as follows: Resistances $R_{1}$ and $R_{2}$, corresponding to the high frequency domain, $R_{1}$ related to the electrolyte resistance and concrete cover resistance, $R_{2}$ to the concrete bulk resistance. A sum of both is attributed to the resistance of the electrolyte including concrete cover and the effects at this interface. The corresponding $\mathrm{C}_{1}$ indicates capacitance of double layers in the bulk matrix in terms of cement paste/aggregate interfacial transition zones (ITZs). The resistance $R_{3}$ is employed to represent the pore solution resistance, the corresponding $C_{2}$ represents the pore network double layer capacitance. The response of the system in the intermediate frequency domain is not indicative of a charge transfer but is due to surface film on the pore walls and $R_{3}$ deals with its ionic resistance. The low frequency domain is normally used to evaluate charge transfer process in combination with mass transport process and resistance $R_{4}$, corresponding to the low frequency range, was used for estimation of the polarization resistance of the embedded steel (12). The corresponding $C_{3}$ is used to determine the interfacial capacitance of the steel surface.

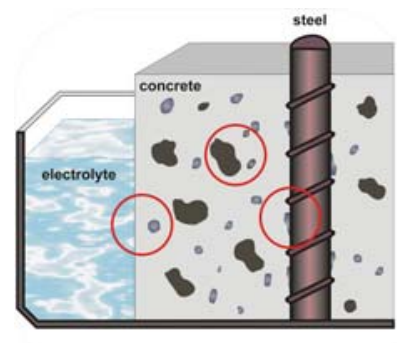

a)

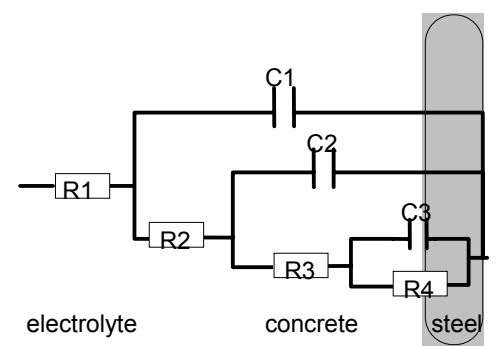

b)

FIGURE 4 Schematic presentation of the reinforced concrete system and different interfaces (circled spots) - (a); Equivalent electrical circuit used in the fitting of the experimental EIS data - (b)

The experimental plots (Figs.5a and b) reveal significantly different response for noncorroding, corroding and protected specimens, attributed to the different technical regimes and electrochemical condition of the steel surface. 

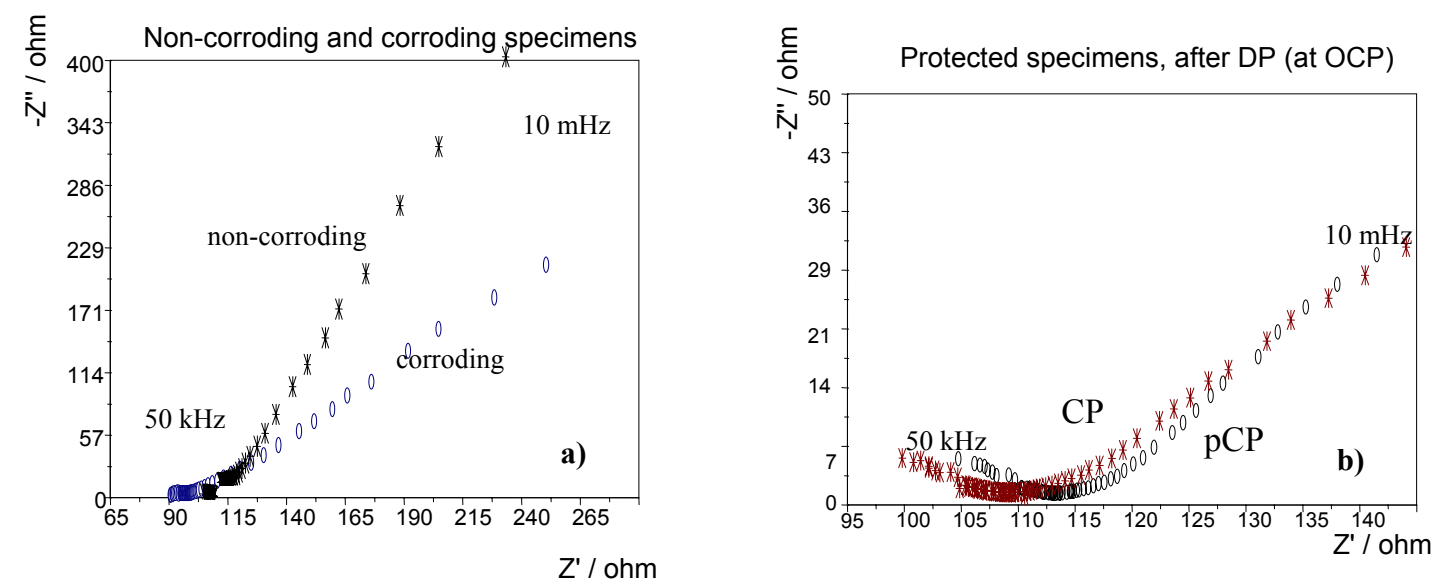

FIGURE 5 Experimental plot (Nyquist format) for non-corroding (control D) and corroding specimens $(\mathrm{N})$ - (a); and for protected specimens $(\mathrm{P})(\mathrm{Cp}$ and pulse $\mathrm{CP})-(\mathrm{b})$

The response and experimental results for the specimens under conventional and pulse CP are almost identical. Summarized data, collected after fitting procedures is presented in Table 1.

TABLE 1. Summarized data from the fitting and simulation procedure of the experimental EIS results

\begin{tabular}{cccccccc}
\hline Cell type & R1 $[\mathbf{O h m}]$ & $\mathbf{C 1}[\mathbf{m F}]$ & $\mathbf{R 2}[\mathbf{O h m}]$ & $\mathbf{C 2}[\mathbf{m F}]$ & $\mathbf{R 3}[\mathbf{O h m}]$ & $\mathbf{C 3}[\mathbf{m F}]$ & $\mathbf{R 4}[\mathbf{k O h m}]$ \\
\hline (N) & 87 & 2.19 & 15.2 & 20.96 & 85.2 & $\mathbf{6 9}$ & $\mathbf{0 . 3 5}$ \\
(P) & 104.4 & 0.001 & 8.82 & 17.64 & 10.9 & $\mathbf{1 2 5}$ & $\mathbf{0 . 0 6}$ \\
(D) & 106.3 & 0.004 & 6.02 & 12.26 & 173.5 & $\mathbf{1 2 . 1 3}$ & $\mathbf{1 . 7 5 8}$ \\
\hline
\end{tabular}

3.2. Linear Polarization Resistance (LPR) and Potentio-Dynamic Polarization (PD). Both methods aimed at deriving the polarization resistance (Rp) of the steel surface in each technical condition, thus evaluating the efficiency of CP technique. Based on the obtained polarization curves, using Tafel and Butler-Volmer equations fit of the Autolab software, the polarization resistance $R p$ was derived. Fig.6b) depicts the experimental PD curves for protected (pulse CP), corroding and non-corroding specimens. The noncorroding specimens exhibit the highest $R p$ and the protected - the lowest. This is expected as the steel surface in the protected specimens, being relatively clean from corrosion products, is activated after depolarization (being at OCP when performing the measurements).

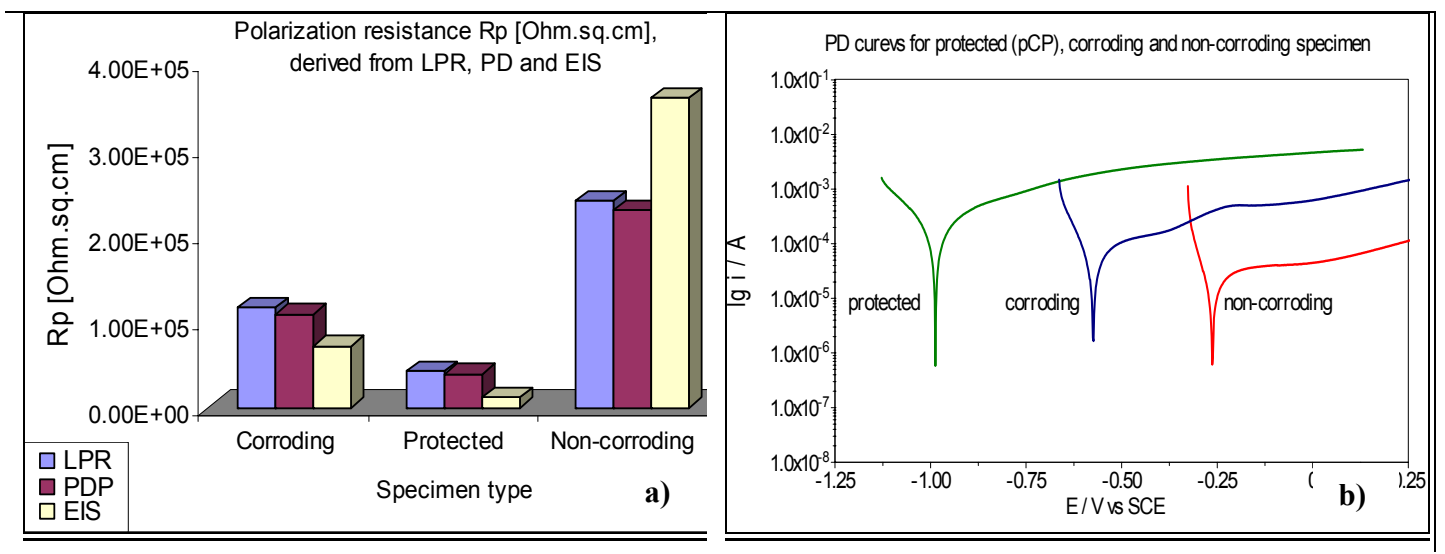

FIGURE 6 Summarized data (Rp values) derived from LPR, PD and EIS - (a) and PD curves for protected, corroding and non-corroding specimens - (b) 
The PD curves reveal mixed anodic/cathodic control for the protected specimens, typical for steel surface in passive (non-active) state, while both corroding and noncorroding specimens exhibit significant anodic control due to the diffusion limitations through the already formed on the surface product layers. The summarized data for $R p$ values derived from the different electrochemical measurements are presented on Fig.6a). The results are consistent for the different measurements, the highest $R p$ (lowest corrosion rate) for the non-corroding (control) specimen and similar corrosion behavior for corroding and protected specimens; even lower values for $R p$ of the protected specimens are recorded by all means. The latter can be explained with the electrochemical behavior of the steel surface after cathodic polarization in two ways: first, the steel surface in the protected specimens, being relatively clean from any product layers and being depolarized for $24 \mathrm{~h}$, becomes in an active state during the time of the measurement. Second existence of calcium carbonate, detected on the steel surface of both corroding and protected specimens, additionally supports active state of the surface in the protected ones, since CP is not running in the time of the measurements. However, the steel surface of the corroding specimen is clean from corrosion products, compared to the corroding surface, as proved from the microstructural investigation (Fig.8 below)

Another consideration that should be taken into account in calculating corrosion rates for example, is the fact that with chloride induced corrosion, the attack is localized, which means that some percentage of the exposed steel is still cathodic. As suggested by (13) a consideration of $10 \%$ anodic regions should be taken into account when calculating the corrosion rate. Hence, the actual corrosion rates for the protected steel surface could be considered as much lower than those calculated on basis of $R p$ values. As the present study aims just visualization and comparing methods with respect to evaluating $\mathrm{CP}$ efficiency, the previously mentioned calculations are not presented. Moreover, as reported in (13), after a prolonged $\mathrm{CP}$, the corrosion rates for the protected steel (being considered as bare steel) are significantly higher (lower $R p$ values respectively) than the original passive corrosion rate. This is most likely as result of oxygen depletion, increased $\mathrm{pH}$ because of oxygen reduction and water hydrolysis on the steel/paste interface (14). With respect to the last, alkaline attack is also not excluded, taking into consideration the eventually high $\mathrm{pH}$ (about 14) and possible formation of $\mathrm{HFeO}_{2}{ }^{-}$ions at the steel/paste interface (13).

3.3. Microstructural investigation. The microstructural investigation was performed in terms of evaluation the concrete material (using the series of concrete prisms) and in terms of steel surface morphology (using the reinforcement from the series of concrete cylinders), aiming at retrieving information for structural and product layers alterations in each technical condition - no-current, under conventional $\mathrm{CP}$ and under pulse $\mathrm{CP}$ conditions. For the bulk concrete matrix, analyzing BSE images ( 0.317 micron / pixel), allowed deriving of structural parameters as porosity, pore size distribution and interconnectivity at different levels of interfaces in the systems under study. 

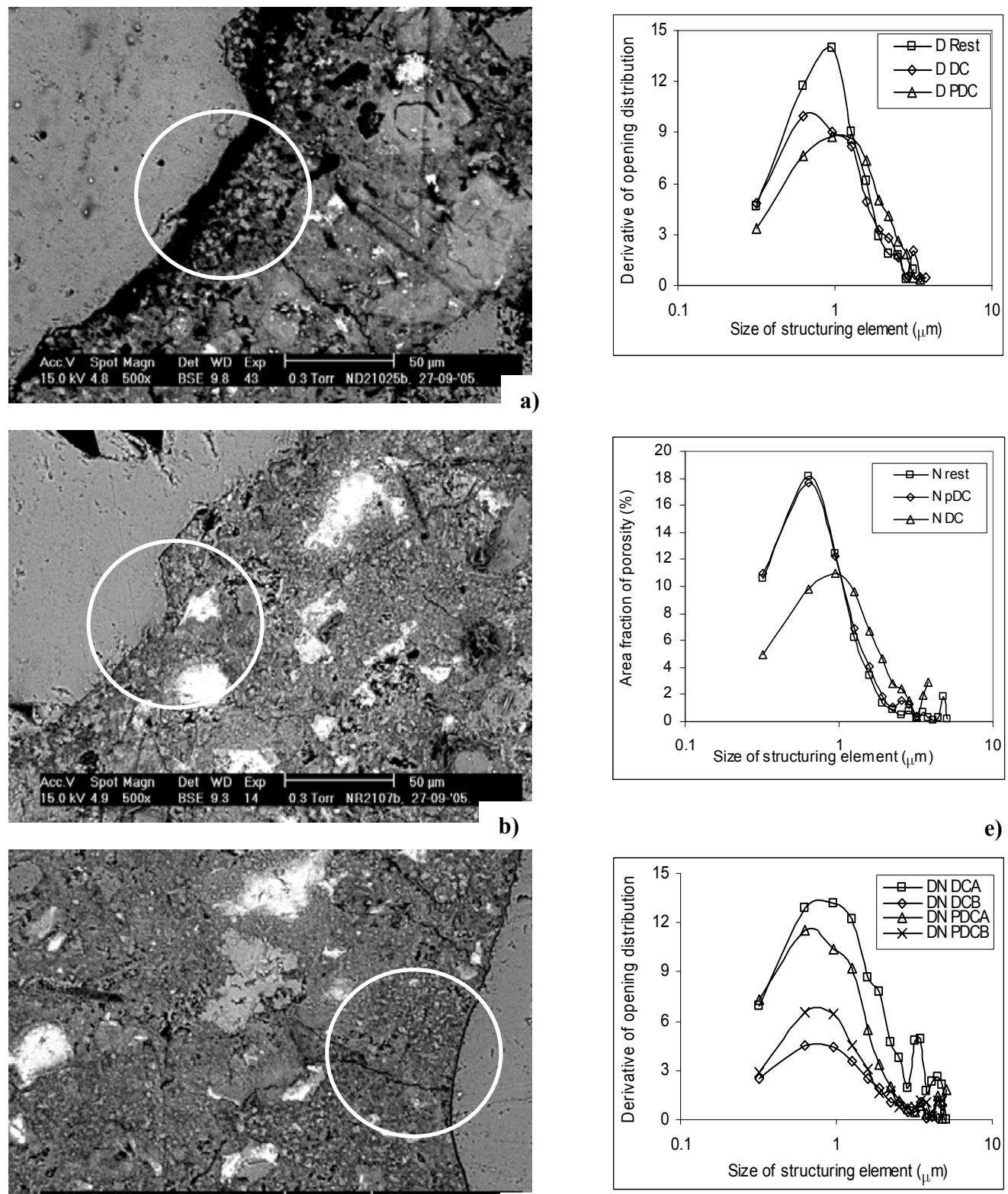

f)

FIGURE 7 (a) to (c): The interfacial transition zone (ITZ) between cement paste and aggregate in specimens N under steady DC conditions - (a), in no current conditions - (b) and pulse DC current conditions - (c) (the SEM image magnification is 1000x) and (d) to (f): Pore size distribution and critical pore size (peak of the curves in the figure) for specimen D (d), N (e) and DN (f) under various conditions, no-current (denoted as 'rest'), pulse (denoted as PDC), and steady (denoted as DC) current, respectively.

DC current causes an obvious decrease in total porosity and coarser pore structure for both containing chloride $(\mathrm{N})$ and free of chloride (D) specimens (Figs.7d,e) while the pulse DC leads to negligible alterations in the pore structure, whereas the el. resistivity is maintained at lower level compared to DC (Fig.2). In DN specimens (half-to-half the mixture of $\mathrm{D}$ and $\mathrm{N}$ ) the steady DC yields higher extent of structural heterogeneity compared to pulse condition (Fig.7f) and higher detrimental effect on the ITZs cement paste/aggregate. As mentioned and hereby illustrated - (Figs.7a-c), the main negative effect of steady DC in terms of concrete microstructure is enlarging the gap between cement paste and aggregate in the ITZs, thus yielding micro-cracks, while in contrast, the pulse DC has negligible influence (Fig.7c) on the bulk concrete matrix. With respect to 
microstructural observations of the steel surface, Fig. 8 depicts the micrographs of corroding and protected specimens. It is evident, that the corroding specimen exhibits severe localized corrosion (pitting) due to the chloride environment, while in the protected specimen, pits were probably initiated (as CP was applied at 60 days of cement hydration) but did not propagate as the steel surface was protected. Corrosion products were not observed in the pits and on the global surface of the protected specimens.
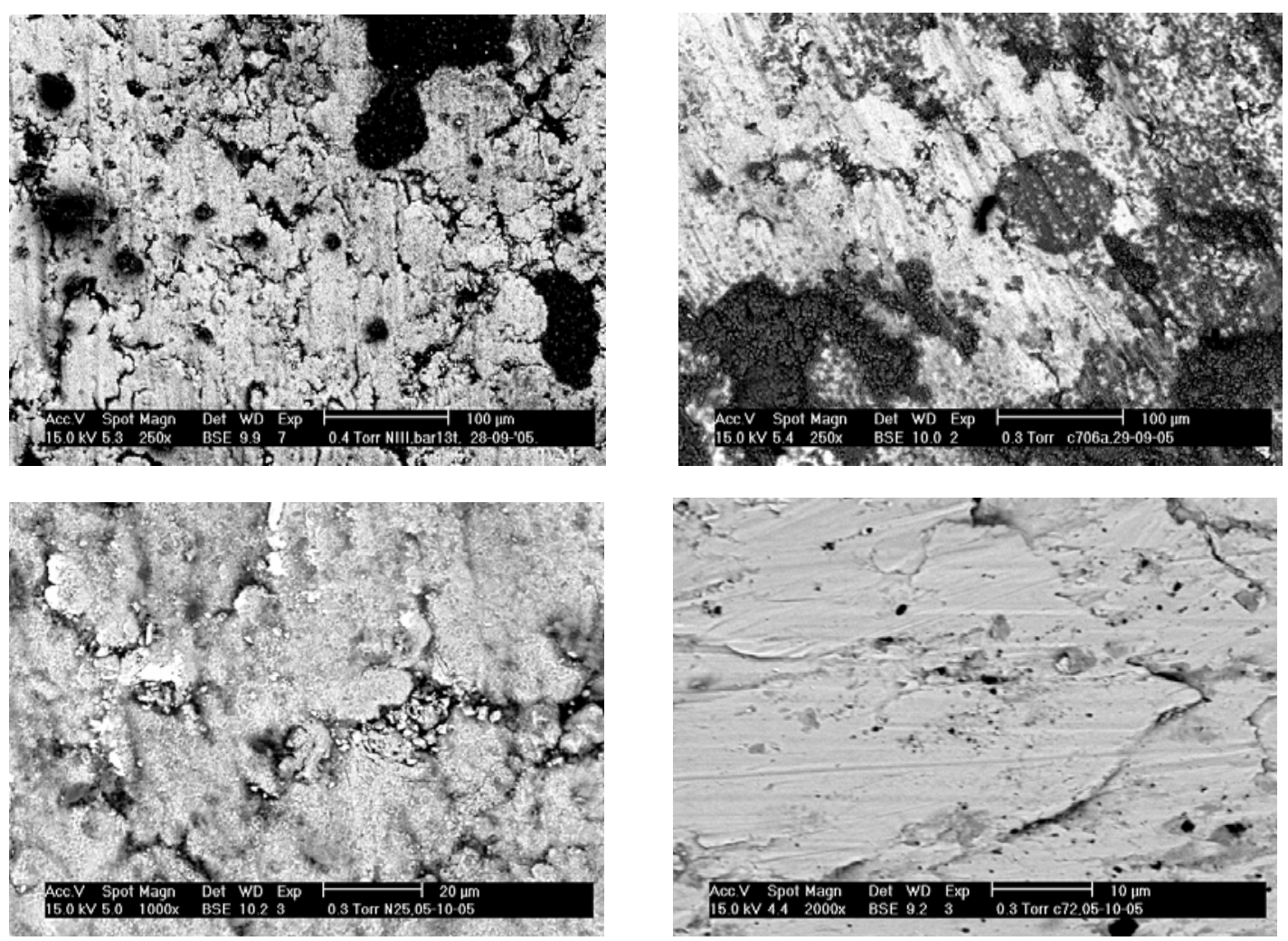

FIGURE 8 Morphological observation of the steel surface in corroding (right column) and protected (left column) specimens at magnification of 250x (overview) and 1000x for the corroding and 2000x for the protected specimens.

\section{CONCLUSIONS}

In conclusion, this contribution explores the feasibility and efficiency of Pulse CP applied to reinforced concrete and its superiorities over conventional $\mathrm{CP}$ by investigating the effects of pulse and steady current on material structure, electrical properties of concrete specimens and electrochemical behavior of steel reinforcement. The combination of conventional monitoring techniques and fundamental approaches leads to better understanding of the involved processes. The research reveals that the pulse current is less detrimental to concrete microstructure and beneficial for electrical properties and ion transport mechanisms. A steady current (as normally used in conventional ICCP applications) tends to bring about unfavorable modifications of the material structure both in the bulk (reducing porosity) and in the interfacial transition zone (enlarging the gap at aggregate surface) to a significant extent, leading to a high level of structural heterogeneity of the materials. With respect to steel surface condition, the research proves that pulse $\mathrm{CP}$ is able to protect the steel reinforcement by achieving sufficient cathodic polarization, while minimizing conditions for unfavorable modifications of the bulk material on micro-level. The additional beneficial effects in 
pulse $\mathrm{CP}$ regime with regard to the material microstructure, electrical properties and ion transport, account for better global performance of $\mathrm{CP}$ protected reinforced concrete structures, hence the pulse CP would be a cost-effective technique for corrosion prevention and protection.

\section{REFERENCES}

1. P. Pedeferri, Constr.Build.Mater., 10, 391 (1996).

2. A.M. Hassanein, G.K.Glass and N.R Buenfeld, Cem.Concr.Comp., 24, 159 (2002).

3. D.A.Koleva, J.Hu and A.L.A.Fraaij, in WIT Press (UK), 48, 87 (2005)

4. J.J. Chang, Cem.Concr. Res., 32, 657 (2002)

5. M. Siegwart, J.F. Lyness and B.J. McFarland, Cem.Concr.Res., 33, 1211 (2003)

6. K. Ishii, H. Seki, T. Fukute and K. Ikwa, Constr.Build. Mater., 12, 125 (1998)

7. K. Takewaka, Corr.Sci., 35, 1617 (1993)

8. B.Reddy, G.K.Glass, P.J.Lim and N.R. Buenfeld, Cem.Concr.Comp., 24, 1 (2002)

9. M.F.Montemor, A.M.P.Simoes and M.G.S.Ferreira, Cem.Concr.Comp., 25 (2003)

10. A.A. Sagues, S.C. Kranc and E.I. Moreno, Corr.Science, 37, 1097 (1995).

11. D.A.Koleva, A.L.A.Fraaij, J.Hu and N.Boshkov, $208^{\text {th }}$ Meeting of ECS, paper 242 Quebec (2005)

12. C.Andrade , L.Soler, C.Alonso, R.X.Novoa and M.Keddam, Corr.Sci.,37, 2013 (1995)

13. T.D.Marcotte, C.M.Hansson and B.B.Hope, Cem.Concr.Res., 29, 1555 (1999)

14. W.K. Green, S.B. Lyon and J.D.Scantlebury, Corr.Sci., 35 (1993) 